\title{
Nonlinearity Mitigation through Optical Phase Conjugation in a Deployed Fibre Link with Full Bandwidth Utilization
}

\author{
S. Yoshima ${ }^{(1,2)}$, Y. Sun ${ }^{(1)}$, K. R. H. Bottrill ${ }^{(1)}$, F. Parmigiani ${ }^{(1)}$, P. Petropoulos ${ }^{(1)}$, and D. J. Richardson ${ }^{(1)}$ \\ (1) Optoelectronics Research Centre, University of Southampton, Southampton, SO17 1BJ, UK. \\ (2) Information Technology R\&D Center, Mitsubishi Electric Corporation, 5-1-1 Ofuna, Kamakura, \\ Kanagawa 247-8501, Japan.
}

s.yoshima@soton.ac.uk

\begin{abstract}
We present a trial of nonlinear impairment mitigation of 16-QAM WDM signals through midlink optical phase conjugation in transmission over an installed fibre link. Signal bandwidth is efficiently reused, avoiding the typical loss of half the spectral band during phase-conjugation.
\end{abstract}

\section{Introduction}

Advanced modulation formats such as 16-QAM are promising candidates to meet the demand for high capacity in future long haul optical transmission systems. As compared to less complex modulation formats (BPSK and QPSK), 16-QAM signals require higher launched powers for the same transmission length due to higher OSNR requirements at the receiver. Nevertheless, fibre Kerr nonlinearity limits the maximum power launched into a given fibre link and, thus, the received OSNR ${ }^{1}$. In order to mitigate the fibre nonlinearity, several approaches have been investigated including: digital back propagation ${ }^{2}$, phase sensitive amplification $^{3}$, phase-conjugated twin waves ${ }^{4}$, and optical phase conjugation $(\mathrm{OPC})^{5-7}$. Amongst these, OPC at the middle of the transmission link has gained some momentum in recent years due to the good performance achieved for relatively simple systems, its modulation format transparency and simultaneous compensation capability for WDM signals. However, the demonstrations so far reported require use of twice the bandwidth of the signal band, as space is usually left free for the phase conjugated signals (idlers) generated in the OPC (idler band) to occupy, thus reducing by half the total transmission capacity. Moreover, most OPC demonstrations have been carried out in laboratory environments, where nearly ideal system designs (Raman amplification and properly designed dispersion shifted fibres) could be exploited to achieve optimum nonlinear compensation $^{5,6}$.

In this paper, we experimentally investigate OPC-based fibre nonlinearity mitigation in a field installed, amplified, standard single mode fibre (SMF) link of about $400 \mathrm{~km}$ length. To retain use of the full transmission band, we demultiplex two sub-bands (populated with 16-QAM signals), B1 and B2, which lie symmetrically either side of the centre of spectral inversion, and perform polarization insensitive $\mathrm{OPC}^{8}$ upon them in two counter-propagating modes of the same highly nonlinear fibre (HNLF) (obviating the need for additional nonlinear media) while using the same two pumps in both directions. The newly formed conjugates $\left(\mathrm{B} 1^{*}\right.$ and $\left.\mathrm{B} 2^{*}\right)$, are remultiplexed and transmitted onwards, resulting in an effective doubling of spectral efficiency over comparable schemes ${ }^{9}$. We study the performance in terms of signal quality factor (Qfactor) with and without OPC as the number of transmitted signals is increased.

\section{Experimental setup}

Figure 1(a) shows the experimental setup of the installed transmission link (part of the UK's Aurora2 network) and the OPC, set at the middle point of the transmission line. The transmitter, OPC, and receiver were located at Southampton, while the repeaters, including EDFAs and dispersion compensation modules (DCMs) were located at both Southampton and Reading. The field-installed fibre was standard SMF (ITU-T G.652d). The total transmission length was about $400 \mathrm{~km}$, consisting of two round trips, the first $180 \mathrm{~km}$ (two $\times 90 \mathrm{~km}$ ) long and the second $220 \mathrm{~km}$ (two $\times 110 \mathrm{~km}$ ) long. The second round trip was $40 \mathrm{~km}$ longer than the first, simply due to the fibre link configuration. It is worth noting that while the performance of OPC is maximized under the condition of symmetric transmission length as well as power profile $^{10}$, it might be difficult to set the OPC at the exact mid-point of deployed networks, as was the reality for our specific case.

The two bands, B1 and B2 were each populated with three $10 \mathrm{Gbaud}$, 16-QAM signals, lying on a $50 \mathrm{GHz}$ grid around centre wavelengths of $1551.72 \mathrm{~nm}$ and $1555.75 \mathrm{~nm}$, respectively. The signals are named such that B1 contains signals S1-S3 and B2 contains signals S4-S6. After characterisation of the system with these signals alone, an additional band (with similar contents to B1 and B2) was added, centred around $1553.73 \mathrm{~nm}$, along with 


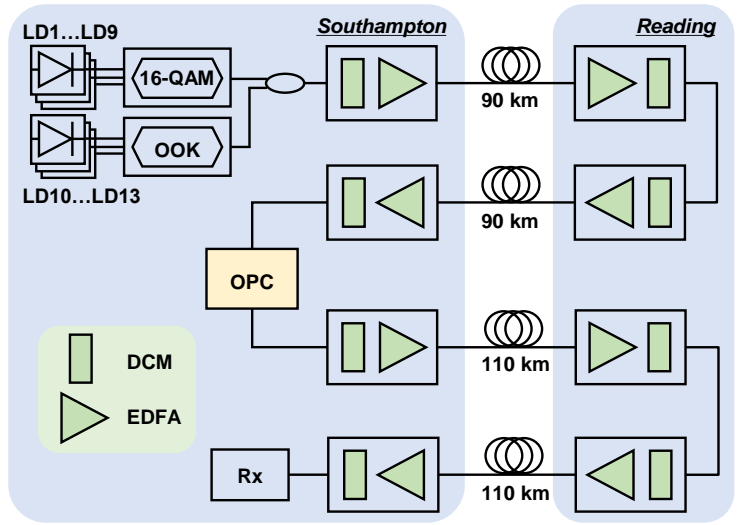

(a) Field trial setup

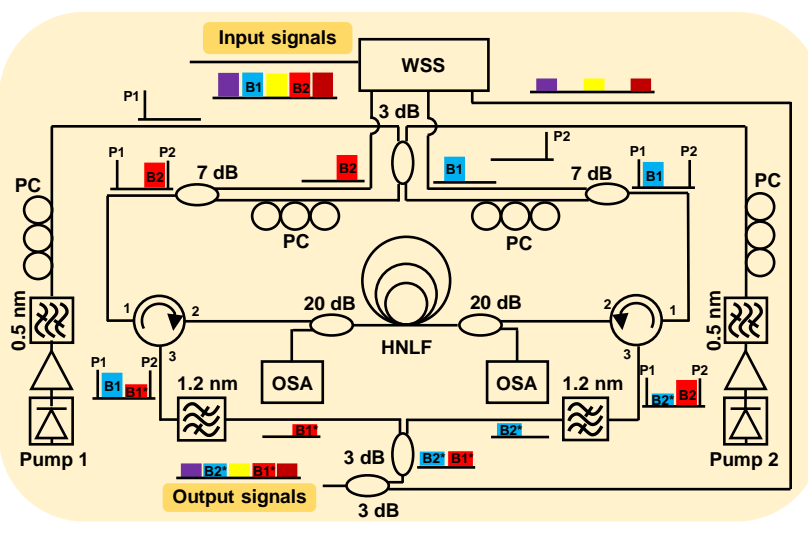

(b) OPC schematic

Fig. 1: Experimental setup.

four 10 Gbaud OOK signals at the following wavelengths: $\quad 1549.32 \mathrm{~nm}, \quad 1550.12 \mathrm{~nm}$, $1557.36 \mathrm{~nm}$, and $1558.17 \mathrm{~nm}$, in order to study the impact of additional WDM channels.

Figure 1(b) shows the experimental setup of the polarization insensitive OPC based on two orthogonal pumps. The incoming signal was input to a WSS for splitting B1, B2, and other channels (through signals) to different outputs. The two CW pumps had linewidths of about $100 \mathrm{kHz}$ at the wavelength of $1558.17 \mathrm{~nm}$ and $1549.32 \mathrm{~nm}$. These two pumps were each amplified to a power of $+22.0 \mathrm{dBm}$ at the HNLF inputs. Optical band-pass filters (OBPFs) of $0.5 \mathrm{~nm}$ were used to suppress amplified spontaneous emission (ASE) noise from the EDFAs. Two polarization controllers (PCs) following the OBPFs were used to make the pumps orthogonally polarized. After combining the two pumps, B1 and B2 were also combined with these two pumps using a $7 \mathrm{~dB}$ coupler and launched into the two ports of the HNLF via circulators. The 300-m long germanium-doped strained HNLF had a nonlinear coefficient of $11.6 /(\mathrm{W}-\mathrm{km})$, a dispersion of $-0.08 \mathrm{ps} /(\mathrm{nm}-\mathrm{km})$, a dispersion slope of $0.018 \mathrm{ps} /\left(\mathrm{nm}^{2}-\mathrm{km}\right)$, and zero-dispersion wavelength of $1555 \mathrm{~nm}$. The conversion efficiency of the OPC was about -2 $\mathrm{dB}$ for both HNLF directions. A typical spectrum of the HNLF output for the B1 band and its corresponding idler $\left(B 1^{*}\right)$ is shown in Fig. 2 .

The generated idlers ( $\mathrm{B} 1^{*}$ and $\mathrm{B} 2^{*}$ bands) were filtered by tuneable filters with a bandwidth of $1.2 \mathrm{~nm}$ and combined with each other and the through signals, as depicted in Fig. 1(b).

At the receiver, the signal was detected by a coherent receiver and a real time scope, using a commercial optical modulation analyser to evaluate the $\mathrm{Q}$-factor. The system was also assessed bypassing the OPC for comparative purposes.

\section{Experimental results and Discussion}

Firstly, we investigated the transmission performance with and without OPC, when only the two B1 and B2 bands were transmitted. Figure 3 shows the Q-factors of the middle channels in each band (S2 and S5, respectively) as a function of launched power per channel for each fibre link after $400 \mathrm{~km}$ transmission. Both Q-factors of 12 and 15 (the respective conjugates of S2 and S5) performed better than without OPC for all launch powers, observing a Q-factor improvement of $0.5 \mathrm{~dB}$ for both idlers and a $1 \mathrm{~dB}$ higher optimum launched power per channel with OPC than without OPC due to the fibre Kerr nonlinearity mitigation. The relatively small improvement may be due to the negative conversion efficiency in the OPC, non-optimum symmetric transmission length and power profile. Similar Q-factor improvements for all remaining channels were measured. This measured performance confirms that our proposed OPC simultaneously mitigates the fibre Kerr nonlinearity for both bands after $400 \mathrm{~km}$ installed fibre transmission while providing twice the usable bandwidth when compared to conventional schemes.

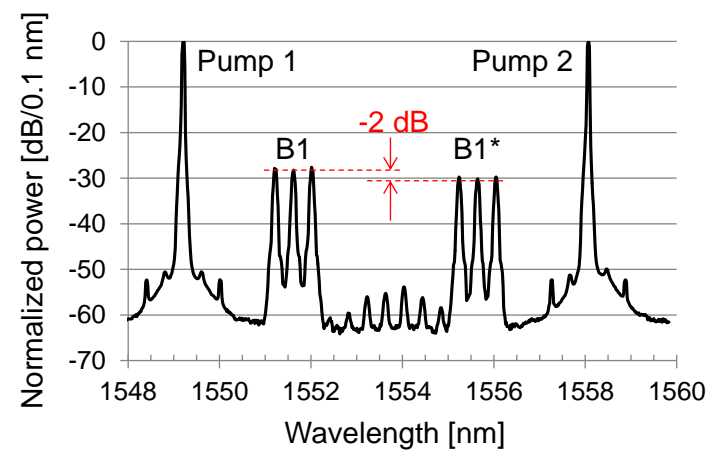

Fig. 2: Typical spectrum ( $0.1 \mathrm{~nm}$ resolution) at the output of the HNLF for $\mathrm{B} 1$ and $\mathrm{B} 1^{*}$ with a conversion efficiency of $-2 \mathrm{~dB}$. 


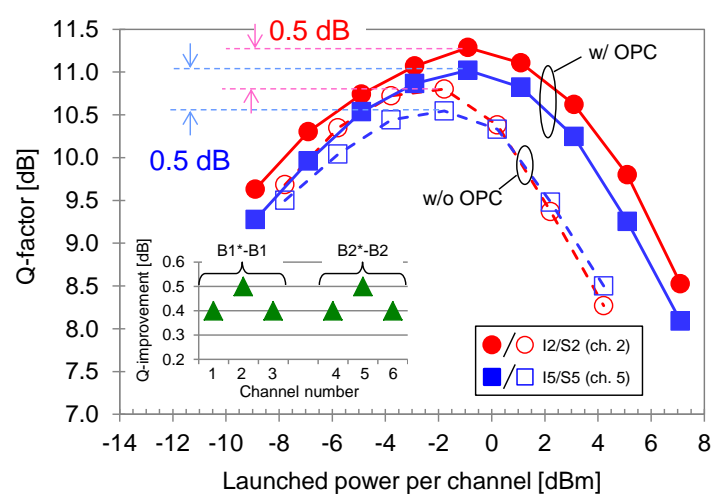

Fig. 3: Q-factors of the middle channels of $B 1$ and $B 2$ (S2 and S5) with and without OPC versus launched power per channel and Q-factor improvements for all channels transmitting only $\mathrm{B} 1$ and $\mathrm{B} 2$ bands.

Furthermore, we investigated the $\mathrm{Q}$-factor improvement as the number of WDM channels was increased. Figure 4 shows the received spectrum after $400 \mathrm{~km}$ transmission, where the extra WDM channels were added to increase nonlinear crosstalk. The Q-factors of S2 and I2, taken as an example, with and without OPC are shown in Fig. 5. The maximum Q-factor with OPC was $11.0 \mathrm{~dB}$, achieving a Q-factor improvement of $0.6 \mathrm{~dB}$. As expected, as the number of WDM channels increased, the optimum launched power per channel was reduced to about $-4 \mathrm{dBm}$ due to the increased nonlinear phase noise. The constellation maps with and without OPC at the launched powers of about -4 and $0 \mathrm{dBm}$ are also reported in Fig. 5. The reduction in phase noise through the use of OPC is visible for all symbols, but is especially noticeable for the outer symbols of the constellation. The results show that OPC improves the performance further when a larger number of WDM channels is present.

\section{Conclusions}

We have proposed and demonstrated doubling of the transmission capacity of an OPC system located in the middle of a $400 \mathrm{~km}$ deployed transmission link. A Q-factor improvement of more than $0.4 \mathrm{~dB}$ was measured for all six 10 Gbaud 16-QAM signals when an OPC was located (close to) the middle of the transmission link. This value could be increased up to $0.6 \mathrm{~dB}$ as the number of WDM channels was increased to 13 , which indicates that OPC may improve the performance further as the number of WDM channels increases even more.

This experiment was performed on the jointly EPSRC/JISC supported NDFIS Aurora2. The work is part of the Photonics Hyperhighway Programme Grant.

\section{References}

[1] R. J. Essiambre et al., "Capacity trends and limits of optical communication networks," Proc. IEEE, Vol. 100,

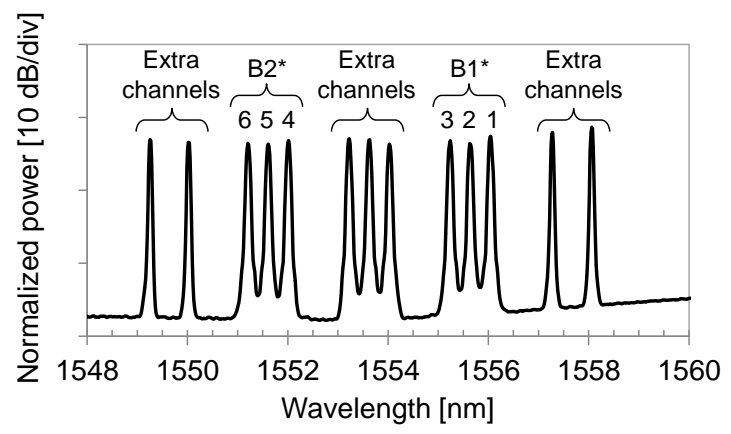

Fig. 4: Received spectrum of idlers including extra WDM channels after $400 \mathrm{~km}$ transmission.

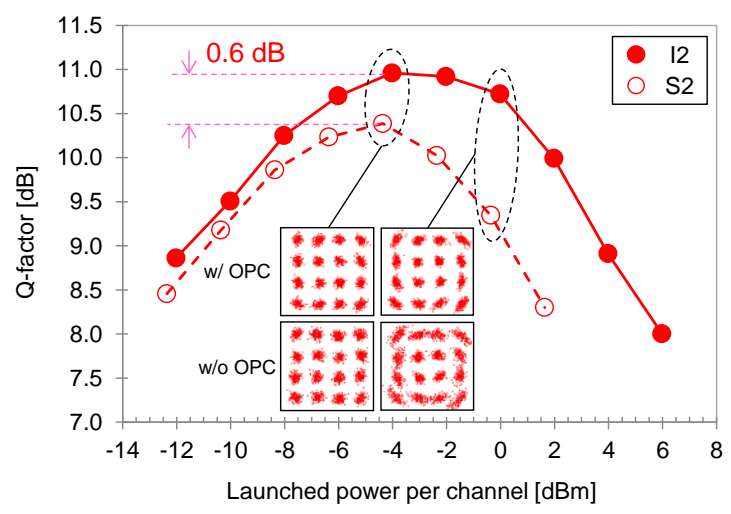

Fig. 5: Q-factors of $S 2$ and 12 versus launched power per channel with extra WDM channels.

p. 1035 (2012).

[2] E. Ip, "Nonlinear compensation using backpropagation for polarization-multiplexed transmission," JLT, Vol. 28, p. 939 (2010).

[3] F. Parmigiani, "Phase sensitive amplifiers and their applications," Proc. OFC, W4C.1 (2015).

[4] X. Liu et al., "Phase-conjugated twin waves for communication beyond the Kerr nonlinearity limit," Nature Photonics, Vol. 7, p. 560 (2013)

[5] I. Sackey et al., "Kerr nonlinearity mitigation: mid-link spectral inversion versus digital backpropagation in 5×28-GBd PDM 16-QAM signal transmission," JLT, Vol. 33, p. 1821 (2015)

[6] K. Solis-Trapala et al., "Transmission optimized impairment mitigation by 12 stage phase conjugation of WDM $24 \times 48 \mathrm{~Gb} / \mathrm{s}$ DP-QPSK signals," Proc. OFC, Th3C.2 (2015).

[7] I. D. Phillips et al., "Exceeding the nonlinear-Shannon limit using Raman laser based amplification and optical phase conjugation," Proc. OFC, M3C.1 (2014).

[8] V. J. F. Rancano et al., "100-GHz grid-aligned multichannel polarization insensitive black-box wavelength converter," JLT, Vol. 32, p. 3027 (2014).

[9] S. Yan et al., "Archon: a function programmable optical interconnect architecture for transparent intra and inter data center SDM/TDM/WDM networking," JLT, Vol. 33, p. 1586 (2015).

[10]K. Solis-Trapala et al., "Signal power asymmetry tolerance of an optical phase conjugation-based nonlinear compensation system," Proc. ECOC, We.2.5.4 (2014). 